\title{
Detection and characterization of early plaque formations by Raman probe spectroscopy and optical coherence tomography: an in vivo study on a rabbit model
}

Christian Matthäus Sebastian Dochow Kokila D. Egodage Bernd F. Romeike Bernhard R. Brehm Jürgen Popp 


\title{
Detection and characterization of early plaque formations by Raman probe spectroscopy and optical coherence tomography: an in vivo study on a rabbit model
}

\author{
Christian Matthäus, ${ }^{a, b, \star}$ Sebastian Dochow, ${ }^{a, b}$ Kokila D. Egodage, ${ }^{a, b}$ Bernd F. Romeike, ${ }^{c}$ Bernhard R. Brehm, \\ and Jürgen Popp ${ }^{a, b}$ \\ aLeibniz Institute of Photonic Technology, Jena, Germany \\ ${ }^{\mathrm{b} F r i e d r i c h-S c h i l l e r-U n i v e r s i t y, ~ I n s t i t u t e ~ o f ~ P h y s i c a l ~ C h e m i s t r y ~ a n d ~ A b b e ~ C e n t e r ~ o f ~ P h o t o n i c s, ~ J e n a, ~ G e r m a n y ~}$ \\ ${ }^{c}$ Friedrich Schiller University, Institute of Pathology, Neuropathology Section, Jena, Germany \\ ${ }^{\mathrm{d} H e r z-N e u r o-C e n t e r ~ B o d e n s e e, ~ C a r d i o l o g y, ~ K r e u z l i n g e n, ~ S w i t z e r l a n d ~}$
}

\begin{abstract}
Intravascular imaging techniques provide detailed specification about plaque appearance and morphology, but cannot deliver information about the biochemical composition of atherosclerotic plaques. As the biochemical composition is related to the plaque type, important aspects such as the risk of a plaque rupture and treatment are still difficult to assess. Currently, various spectroscopic techniques are tested for potential applications for the chemical analysis of plaque depositions. Here, we employ Raman spectroscopy in combination with optical coherence tomography (OCT) for the characterization of plaques on rabbits in vivo. Experiments were carried out on New Zealand white rabbits treated with a fat- and cholesterol-enriched diet, using a Raman probe setup with a 785-nm multimode laser as an excitation source. Subsequently, OCT images were acquired with a swept source at $1305 \pm 55 \mathrm{~nm}$ at $22.6 \mathrm{~mW}$. Raman spectra were recorded from normal regions and regions with early plaque formations. The probe positioning was monitored by x-ray angiography. The spectral information identified plaque depositions consisting of lipids, with triglycerides as the major component. Afterward, OCT images of the spectroscopically investigated areas were obtained. The spectral information correlates well with the observed intravascular morphology and is in good agreement with histology. Raman spectroscopy can provide detailed biochemical specification of atherosclerotic plaques. ( 2018 Society of Photo-Optical Instrumentation Engineers (SPIE) [DOI: 10.1117/1.JBO.23.1.015004]
\end{abstract}

Keywords: Raman spectroscopy; optical coherence tomography; atherosclerosis; fiber probes.

Paper 170358PRR received Jun. 23, 2017; accepted for publication Dec. 15, 2017; published online Jan. 9, 2018.

\section{Introduction}

Over the past 10 years, advances in technologies have opened opportunities to utilize spectroscopic modalities for biological and medical applications. The spectral information obtained by observing the interaction of a sample with electromagnetic radiation is very versatile and can offer qualitative and quantitative characterization of its biochemistry. Among the very popular spectroscopic techniques that can be easily coupled to optical elements are methods based on electronic or vibrational excitations in the visible or infrared (IR) region of the electromagnetic spectrum. Vibrational excitations can be generated by either absorption or inelastic scattering of light, which are the bases of IR/NIR and Raman spectroscopy, respectively. Several spectroscopic techniques have been applied to characterize atherosclerotic plaques in order to obtain information about the biochemical composition of the affected tissue. From post mortem studies, it is well known that the severity of a plaque and its stability are strongly correlated with its biochemical composition. ${ }^{1}$ The identification of vulnerable plaques especially remains one of the most important and challenging aspects in cardiology. Thus, specific information about the

*Address all correspondence to: Christian Matthäus, E-mail: christian matthaeus@leibniz-jena.de composition of a plaque would greatly improve the risk assessment and management. Furthermore, knowledge about the composition can offer new therapeutic and medication strategies. The application of spectroscopic techniques to biological specimens is meanwhile relatively straightforward. The greatest challenge is the direct application in vivo. Currently, there are several indispensable noninvasive and invasive imaging modalities to diagnose atherosclerosis and localize affected areas. Techniques based on computer tomography (CT), such as electron beam CT (EBCT) and multidetector CT (MDCT), can identify calcification levels, lipid cores, and vessel remodeling, but are either too specific (EBCT only detects calcification) or not sensitive enough (MDCT exhibits high negative but low positive predictive values). ${ }^{2}$ Positron emission tomography can identify inflammation, which is an indicator of vulnerability, but not the only one. ${ }^{2}$ The most common invasive techniques are intravascular ultrasound (IVUS) and optical coherence tomography (OCT). Both catheter-based modalities deliver very precise images of plaque morphologies that can be indicative for certain plaque types. IVUS detects the amplitude of soundwaves and has a penetration depth of several millimeters that allows visualizing the lamina of the vessel wall. The obtained grayscale image can be further interpreted by radiofrequency analysis,

$1083-3668 / 2018 / \$ 25.00$ @ 2018 SPIE 
which can be depicted in a false color image termed as virtual histology (VH-IVUS). ${ }^{3}$ OCT on the other hand is based on interferometry and has a better spatial resolution, but cannot penetrate much more than 1 to $2 \mathrm{~mm}$. IVUS and OCT are complementary to each other and hybrid modalities are in development. ${ }^{4,5}$ In any case, both modalities deliver grayscale images that deliver rather unprecise information about the biochemical composition of the plaque and require a very high amount of long time empirical expertise from the point of inspection. Spectroscopic techniques would, therefore, be an ideal tool to complement the imaging information. Among the first methods, near-infrared spectroscopy (NIRS) has been introduced that is especially sensitive to the identification of lipid pools. ${ }^{6,7}$ NIRS probes for overtones and combinations of molecular vibrations and can contrast lipid molecules such as cholesterol or triglycerides against the collagen of the vessel wall. It is not the most sensitive technique with little penetration, but offers molecular information of especially bulk material without interference from water. In combination with sophisticated data analysis, pullback chemometric images can be generated. To support the plaque visualization, NIRS is commercialized in combination with IVUS and has very recently been successfully applied in clinical studies. ${ }^{8,9}$ Another promising spectroscopic approach is fluorescence lifetime imaging (FLIM), which is based on the different, molecule specific, decay time of laser-induced autofluorescence. The technique can distinguish different plaque components and has been applied ex vivo on open vessel carotenoid plaques by an en face illumination and in vivo in combination with ultrasound. ${ }^{10-13}$

Along with the development of NIRS for the characterization of plaque compositions, the potential of Raman spectroscopy has been investigated. As a vibrational spectroscopic technique, it is closely related to the IR techniques. The first Raman studies of atherosclerotic tissue were reported at about the same time as the NIR data. ${ }^{14}$ Meanwhile, there have been a few attempts in applying the technique under in vivo conditions. ${ }^{15,16}$ Several probe designs have been tested on different animal models. Because molecular vibrations and not overtones are probed, the obtained spectra contain much more information and are more sensitive with respect to the overall composition of the plaques. Main components such as lipids and calcifications can be readily identified. Raman-probe spectroscopy has been combined with other spectroscopic modalities as for instance intrinsic fluorescence and diffuse reflectance spectroscopy to evaluate the potential of multimodal spectroscopy for the detection plaques with a particular emphasis on the identification of vulnerable plaque sites. ${ }^{17}$ Recently, the potential of intravascular Raman spectroscopy has been demonstrated in vivo on a rabbit model. ${ }^{18}$ Main aspects in the probe design can now be addressed and measuring conditions, such as the incoming laser power and acquisition time, are feasible for in vivo conditions. The greatest challenge at the moment is the development of Raman probes that have geometrical parameters that are compatible for applications in humans. In order to overlay the obtained spectral information with plaque morphology, it will be ideal to combine such a probe with an intravascular imaging modality such as IVUS or OCT. Since the signal generation in both OCT and Raman spectroscopy is realized by laser radiation, the combination of the latter is technically much more suitable. In order to test the feasibility of OCT and Raman spectroscopy under similar conditions, we applied both technologies to a rabbit model and correlated the spectral information with the OCT images.

\section{Methods}

\subsection{Raman Probe Design and Data Acquisition}

The employed Raman probe (EM Vision, Loxahatchee, Florida) consists of one multimode excitation waveguide surrounded by 12 multimode collection fibers with $125 \mu \mathrm{m}$ diameter and $100 \mu \mathrm{m}$ as the core diameter. The numerical aperture of the excitation fiber was 0.22 . These are packed and adjusted in a steel guidance tube resulting in a total outer probe diameter of $1 \mathrm{~mm}$. Both light paths are equipped with edge filters, embedded in front of the fibers and stabilized by the guidance tube and guidance rods. Raman excitation was provided by an Invictus multimode laser with an FC/PC coupling. To meet the requirements of the SMA input of the excitation path connector, an SMA-FC/PC adapter was used. As the excitation wavelength, 785-nm $\mathrm{cw}$ laser was used with a fiber-coupled power of $100 \mathrm{~mW}$. Due to coupling losses, the excitation power at the sample is about $60 \mathrm{~mW}$. The acquisition time was set between 1 and $10 \mathrm{~s}$. At the output of the probe, the 12 collection waveguides are stacked in one line inside an SMA connector. The fiber connector was adjusted parallel to the illumination slit of the spectrometer and was projected to the CCD of the RXN1 spectrometer (Kaiser optical systems) by a holographic transmission grating, which covers the full spectral region from 0 to $3500 \mathrm{~cm}^{-1}$. Binning was adjusted to minimize CCD noise and background effects. A spectrometer wavelength calibration, as well as an intensity correction for changing quantum efficiencies of the CCD was done after moving the spectrometer to the hospital. The laser wavelength was calibrated by cyclohexane after the warm up procedure directly before surgery. The whole system was set on a movable table to be flexible during the operation.

\subsection{OCT Imaging}

For OCT imaging, an ILUMIEN Optis system (St. Jude Medical) with a swept source at $1305 \pm 55 \mathrm{~nm}$ at $22.6 \mathrm{~mW}$ was used, which enabled an A-scan depth of $7 \mathrm{~mm}$ in air and $4.83 \mathrm{~mm}$ in the used contrast agent. Therefore, the imaging depth of OCT in tissue should be $7 \mathrm{~mm} / n$ _tissue, $n: 1.33$ to 1.55. The system had an A-scan repetition rate of $90 \mathrm{kHz}$ that allows a frame rate of 180 images per second. As catheter, the corresponding C7 Dragonfly (St. Jude Medical) was used. The catheter was inserted through a $6 \mathrm{~F}$ introducer (B. Braun, Melsungen, Germany) attached to a guidewire (Terumo, Tokyo, Japan), which was advanced into the descending aorta. Before the OCT imaging, the contrast agent was manually injected into the side port of the imaging catheter. The software of the ILUMEN system recognized the purging with the contrast agent and automatically started a pullback sequence with the predetermined length of $40 \mathrm{~mm}$. All data were saved to the ILUMEN system and reviewed with the help of a cardiologist.

\subsection{OCT-Raman In Vivo Experiments}

\subsubsection{Animals}

Adult male New Zealand White rabbits (Harlan) were studied. To generate atherosclerotic changes of the vascular system, rabbits were fed with a $0.5 \%$ cholesterol diet for different periods $(7 / 10 \pm 1$ weeks $)$. 


\subsubsection{Angiography}

Rabbits weighing 3.25 to $3.58 \mathrm{~kg}$ were anesthetized by intravenous injections of ketamine 2 to $8 \mathrm{mg} / \mathrm{kg}$ (Ketamin "Gräub" 10\%, Albrecht, Aulendorf; Germany) and xylazine 0.5 to $1 \mathrm{mg} / \mathrm{kg}$ (Rompun Trockensubstanz, Bayer, Leverkusen, Germany). The maintenance of the anesthesia was realized through intravenous administration of ketamine $4 \mathrm{mg}$ and xylazine $1 \mathrm{mg}$ via a marginal ear vein. Via cervical incision, the right common carotid artery was prepared and the distal end was ligated. After small arteriotomy, a $6 \mathrm{~F}$ introducer (B. Braun, Melsungen, Germany) was inserted, followed by the insertion of a guidewire (Terumo, Tokyo, Japan), which was advanced into the descending aorta. Via a $6 \mathrm{~F}$ catheter (Cordis, Langenfeld, Germany) contrast medium (Visipaque, GE Healthcare Buchler, München, Germany) was injected to obtain an angiogram of the complete aorta. Subsequently, the Dragonfly catheter was introduced and several C-scans over the whole scan range were done. In the next step, the catheter was withdrawn, the Raman-probe advanced until the aortic bifurcation and was slowly removed to generate Raman-data on defined aortic places (Fig. 2). At an acquisition time of 1 to $10 \mathrm{~s}$ per point spectrum, the total experiment time per animal was not longer than 5 min for the Raman data collection.

\subsubsection{Scarification/pressure perfusion}

After the data acquisition, the rabbits were euthanized by an overdose of the anesthetic solution. With a vertical thoracic to abdominal incision, the aorta was prepared and a perfusion cannula was inserted into the descending aorta. A 150-ml mixture of $10 \%$ hydroxyethyl starch (Fresenius Kabi Deutschland $\mathrm{GmbH}$, Bad Homburg, Germany) and $1 \%$ procaine in a ratio of 10 to 1 was used for vessel-fixation, followed by a $0.9 \%$ saline (Fresenius Kabi Deutschland GmbH, Bad Homburg, Germany) pressure perfusion over $15 \mathrm{~min}$ at $100 \mathrm{mmHg}$. The complete aorta was excised and preserved in 5\% formalin solution (Oscar Fischer GmbH, Saarbrücken, Germany) for preparation of the samples for histopathological staining.

All animal tests for the studies were approved by the Thüringer Landesamt für Verbraucherschutz (TLV), Germany.

\section{Results}

After the application of the catheter through the carotid artery of the rabbit, the Raman probe was inserted and positioned at the lower aorta just before the bifurcation of the iliac arteries. Raman measurements were started within the abdominal aorta at illumination times between 1 and $3 \mathrm{~s}$. Spectra were taken approximately every $0.5 \mathrm{~cm}$. During the measurements, the body of the rabbit was constantly monitored by x-ray to record the exact position of the Raman probe. The appearance of the spectra was inspected for Raman bands and band patterns characteristic for lipids. Principally, spectral patterns of lipids, such as cholesterol, cholesterol esters or triglycerides can be easily distinguished from spectra of the vessel wall, which mainly consists of collagen and, therefore, exhibits spectral features associated with molecular vibrations of the protein backbones and typical functional groups. During the experiment, the Raman probe was carefully withdrawn through the abdominal and thoracic aorta into the aortic arch. The first noticeable spectral features of lipids were found within the upper part of the aorta above the heart of the rabbit. Figure 1 shows the spectrum of a normal part of the aorta from the

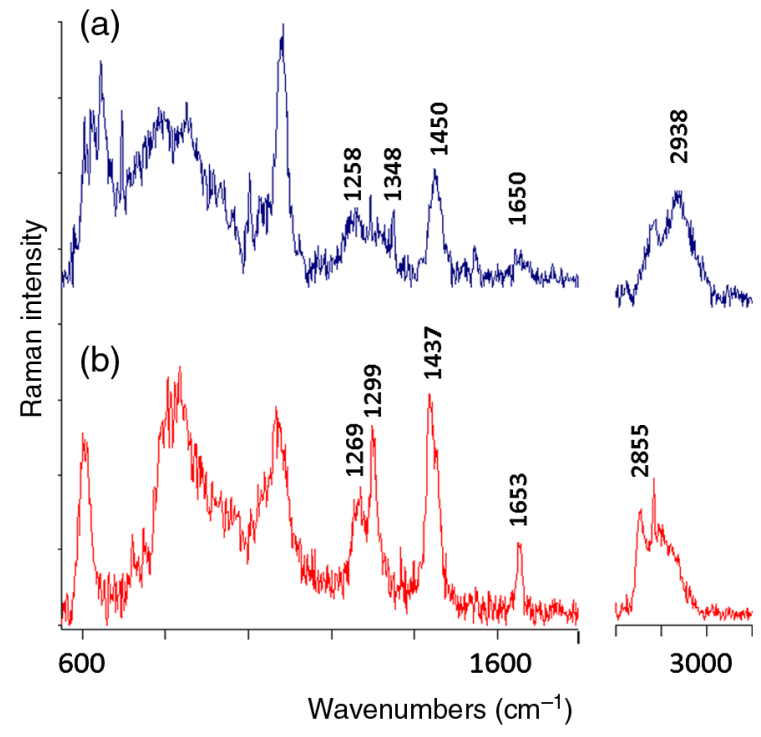

Fig. 1 Raman spectra of an aorta position without plaque depositions (a), plotted in blue, and with lipid depositions (b), plotted in red. Spectrum (a) shows the typical spectral features of proteins, reflecting the protein/collagen composition of the arterial wall. Spectrum (b) exhibits all spectral characteristics typical for lipids.

abdominal region in comparison with a spectrum from the region close to the aortic arch. The Raman spectrum of the normal vessel shows the typical characteristics of vibrational bands of proteins, whereas the spectrum of the vessel of the upper part of the aorta exhibits Raman bands typical for lipids. Easy to notice are enhanced band intensities in the region between 2800 and $3100 \mathrm{~cm}^{-1}$, as well as within the lower spectral region between 900 and $1800 \mathrm{~cm}^{-1}$. All Raman bands can be clearly assigned to either proteins or lipids. The spectral features of arterial walls and lipids have been previously described in detail. $^{19}$

After the recording of the Raman spectra of normal and abnormal appearance, the Raman probe was exchanged with an OCT probe. Again assisted by the guidance of the catheter, the probe was moved to the position where the Raman spectra with lipid features were measured. The associated OCT images are shown in Fig. 2. Figures 2(A)-2(C) depict horizontal OCT images of different positions within the thoracic aorta that show rough deformations at the walls of the vessel. The diameter of the aorta is about $3.5 \mathrm{~mm}$ and the penetration of the OCT is about $0.75 \mathrm{~cm}$, which can be estimated by the obtained OCT images shown in Fig. 2. The white arrows indicate clearly noticeable irregularities of the inner vessel wall, which are on the order of several $100 \mu \mathrm{m}$. Figure 2(D) shows a vertical profile of the aorta. The positons of the horizontal images are indicated between 15 and $20 \mathrm{~mm}$. The region that was imaged by OCT is $3 \mathrm{~cm}$ long, before the probe enters the end of the catheter. The aorta can be generally characterized as normal with a few positions of early plaque formations.

After the acquisition of the Raman and OCT data, the animal was euthanized, the aorta perfused with a physiological buffer solution and subsequently fixed in formalin for routine histology. The thoracic fraction of the aorta was dissected for histopathological inspection. Figure 3(a) shows an enlarged area of the OCT Fig. 2(A) as indicated by the inset in comparison with an elastic van Gieson (EvG) stained section from the same region, shown in Fig. 2(B). Although precisely overlapping 


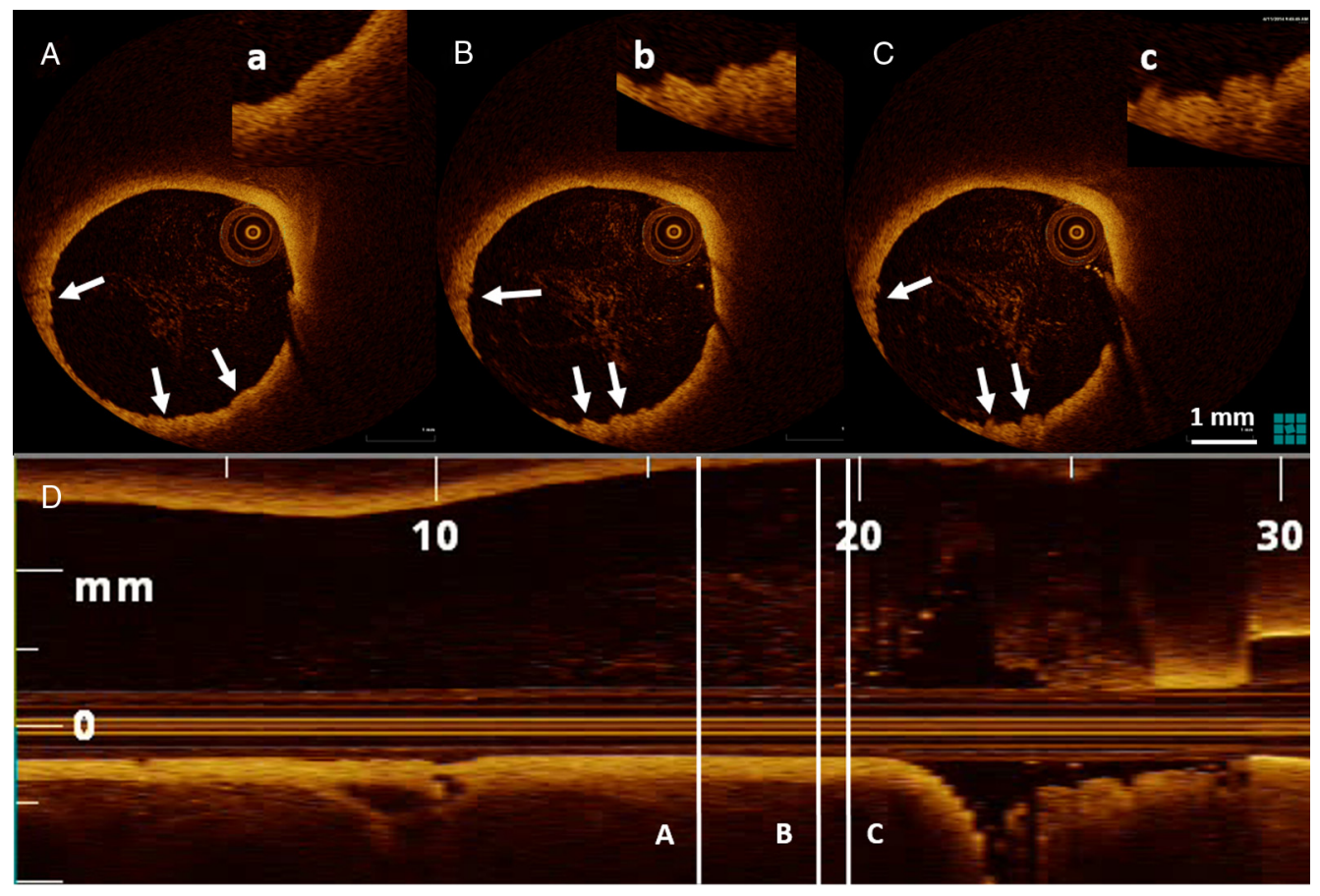

Fig. 2 OCT images of a rabbit aorta with early plaque formations indicated by the white arrows in (A-C) (a-c zoomed in). (D) is a vertical image of the aorta. The positions of the horizontal images are indicated by the white lines.
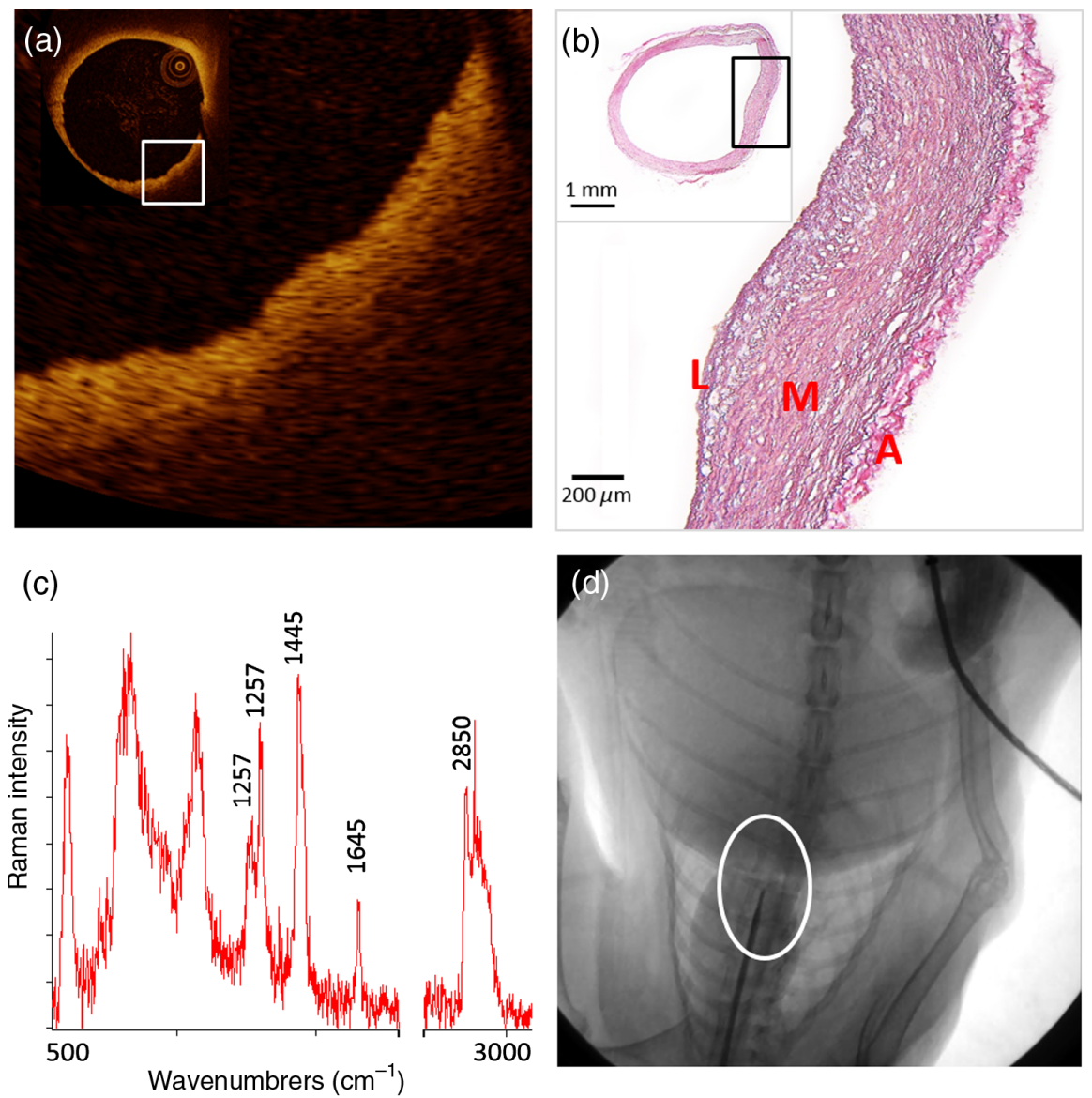

Fig. 3 Enlarged OCT image (a) from the section shown in Fig. 2(A) in comparison with an EvG stain (b) of a section from the same region; $L$, lumen of artery; $M$, muscularis media; and $A$, adventitia. Graph (c) shows a Raman spectrum with characteristic lipid bands collected within the region where the OCT image was obtained. The position of the Raman probe is shown in (d). 
OCT and microscopic images are difficult to obtain, the similarity in morphology is obvious. Clearly noticeable is a swollen intima, which is about 100 to $200 \mu \mathrm{m}$ thick. The magnification shows optically empty vacuoles, indicating former lipid depositions. Figure 3(c) shows a Raman spectrum measured in that region with the typical spectroscopic features associated with lipids. The $\mathrm{CH}$ stretching vibrations between 2800 and $3100 \mathrm{~cm}^{-1}$ are dominated by the symmetric methyl stretches at $2850 \mathrm{~cm}^{-1}$. The bands below $1800 \mathrm{~cm}^{-1}$ can be assigned to the $\mathrm{C}=\mathrm{C}$ stretching vibrations of unsaturated lipids at $1640 \mathrm{~cm}^{-1}, \mathrm{CH}_{2}$ scissoring vibrations at $1440 \mathrm{~cm}^{-1}$, and $\mathrm{CH}$ deformations at saturated and unsaturated positions between 1150 and $1300 \mathrm{~cm}^{-1}$. The lower wavenumber region is dominated by background scattering generated by the quartz fibers of the probe. The obtained $S / N$ ratio reveals most of the Raman bands typical for lipids. The overall spectral appearances indicate plaque depositions mainly consisting of triglycerides.

\section{Discussion}

It has been demonstrated that the acquisition of OCT images and Raman spectroscopic data is generally possible. The most important outcome of the application in vivo was that both methodologies can be applied consecutively under the same conditions. The current technology level is sufficiently suitable to detect lipids and potentially to characterize plaque composition by Raman spectroscopy on a New Zealand white rabbit model. As it is a common animal model to study atherosclerosis with similarities to human plaques,${ }^{20}$ the outcome of the experiments describe the possible conditions for in vivo investigations of plaque developments and treatments, such as balloon angioplasty or stent implantation. Second-generation balloons for angioplasty and stents, which are coated with drugs to prevent early restenosis, are currently under development. ${ }^{21-23}$ The efficiency of such drug-coated balloons and drug eluting stents can be investigated by directly monitoring the concentration and long-term release spectroscopically, as the active compounds also exhibit distinct spectroscopic properties. ${ }^{24,25}$

The applied conditions of several seconds of nonfocused laser illumination at $100 \mathrm{~mW}$ within the artery did not lead to any noticeable alterations of the walls of the blood vessels, as confirmed by subsequent histopathological staining. Raman spectroscopy proved to be sensitive enough to detect early plaque formations. Since the scattering cross section of lipids is generally high, lipid depositions can be easily contrasted against normal tissue. To address concentration thresholds for lipids within a tissue matrix is relatively difficult, as the lipids are not dissolved and will always form crystalline aggregates or droplets, varying in size. Schie et al. have investigated ratios of different lipids within lipid droplet organelles and were able to differentiate lipids of different saturation levels down to a few mass percentages. $^{26}$

The obtained data quality was sufficient to assign the major depositions as triglycerides, which was surprising as the animals were treated with a fat- and cholesterol-enriched diet. ${ }^{27}$ Previous results showed depositions dominated by cholesterol and cholesterol esters, but also revealed accumulations of triglycerides. ${ }^{18,28}$ Generally, the animals did not show the expected amounts of plaque formations. The presented results were obtained by inserting the Raman probe first, followed by the OCT imaging pullbacks. In principle, the application order does not influence the results. Here, practical aspects, such as the animals' limited wellbeing, affected by the injected contrast agents for the OCT, were taken into consideration. For studies in human or other in vivo experiments, a reverse order may be more appropriate. Since both methodologies utilize laser light in the NIR region, a probe that combines OCT and Raman spectroscopy is technologically feasible. Because Raman spectroscopy is inherently slower than OCT, at the current state-of-the-art technical development, it cannot be utilized for intravascular imaging as a standalone modality. One of the main challenges will be a coregistration of the two signal types. A combined OCT-Raman fiber would resolve this issue as the two incident laser spots can be overlapping. A combination with IVUS is certainly less feasible as the associated signal generation and detection technologies are completely different. However, signal transduction for NIRS and IVUS also needs to be realized by two different types of fibers. The main reason to combine NIRS with IVUS is that both techniques are of similar speed, so the signal reception can be coregistered. Apart from intravascular vibrational spectroscopy, several other spectroscopic approaches have been suggested and are currently under investigation. Fluorescence-based techniques such as two-photon excitation fluorescence (TPEF) and FLIM are certainly fast enough to record images and have been utilized to characterize atherosclerotic plaques. ${ }^{11,12,29,30}$ TPEF is often combined with other multiphoton processes, such as second harmonic generation and coherent anti-Stokes Raman scattering imaging, a much faster, but also more complicated variant of Raman spectroscopy. ${ }^{28,31-33}$ However, for these multimodal techniques, reliable optical fibers that are applicable for imaging still need to be developed.

\section{Disclosures}

The authors have no relevant financial interests in this article and no potential conflicts of interest to disclose.

\section{Acknowledgments}

Financial support from the Carl Zeiss Foundation is greatly acknowledged.

\section{References}

1. R. Virmani et al., The Vulnerable Atherosclerotic Plaque: Strategies for Diagnosis and Management, Blackwell Publishing, Inc., Malden, Massachusetts (2007).

2. G. Niccoli et al., "Advances in mechanisms, imaging and management of the unstable plaque," Atherosclerosis 233(2), 467-477 (2014).

3. A. König and V. Klauss, "Virtual histology," Heart 93(8), 977-982 (2007).

4. B. Li et al., "Hybrid intravascular ultrasound and optical coherence tomography catheter for imaging of coronary atherosclerosis," Catheterization Cardiovasc. Interventions 81(3), 494-507 (2013).

5. H. Yang et al., "A dual-modality probe utilizing intravascular ultrasound and optical coherence tomography for intravascular imaging applications," IEEE Trans. Ultrason., Ferroelectr., Freq. Control 57(12), 2839-2843 (2010).

6. V. Neumeister et al., "Determination of the cholesterol-collagen ratio of arterial atherosclerotic plaques using near infrared spectroscopy as a possible measure of plaque stability," Atherosclerosis 165(2), 251257 (2002).

7. J. Caplan et al., "Near-infrared spectroscopy for the detection of vulnerable coronary artery plaques," J. Am. Coll. Cardiol. 47(8), C92-C96 (2005).

8. S. Kang et al., "Combined IVUS and NIRS detection of fibroatheromas: histopathological validation in human coronary arteries," JACC: Cardiovasc. Imaging 8(2), 184-194 (2015).

9. K. Huynh, "Imaging: combining IVUS and NIRS improves accuracy of fibroatheroma detection," Nat. Rev. Cardiol. 12(3), 130-130 (2015). 
10. L. Marcua et al., "Detection of rupture-prone atherosclerotic plaques by time-resolved laser-induced fluorescence spectroscopy," Atherosclerosis 204, 156-164 (2009).

11. J. Park et al., "Biochemical characterization of atherosclerotic plaques by endogenous multispectral fluorescence lifetime imaging microscopy," Atherosclerosis 220(2), 394-401 (2012).

12. J. Bec et al., "Multispectral fluorescence lifetime imaging system for intravascular diagnostics with ultrasound guidance: in vivo validation in swine arteries," J. Biophotonics 7(5), 281-285 (2014).

13. H. Yoo et al., "Intra-arterial catheter for simultaneous microstructural and molecular imaging in vivo," Nat. Med. 17(12), 1680-1684 (2011).

14. S. van de Poll et al., "Imaging of atherosclerosis. Raman spectroscopy of atherosclerosis," J. Cardiovasc. Risk 9(5), 255-261 (2002).

15. H. P. Buschman et al., "In vivo determination of the molecular composition of artery wall by intravascular Raman spectroscopy," Anal. Chem. 72(16), 3771-3775 (2000).

16. J. T. Motz et al., "In vivo Raman spectral pathology of human atherosclerosis and vulnerable plaque," J. Biomed. Opt. 11(2), 021003 (2006).

17. O. R. Sćepanović et al., "Multimodal spectroscopy detects features of vulnerable atherosclerotic plaque," J. Biomed. Opt. 16(1), 011009 (2011).

18. C. Matthäus et al., "In vivo characterization of atherosclerotic plaque depositions by Raman-probe spectroscopy and in vitro coherent antistokes Raman scattering microscopic imaging on a rabbit model," Anal. Chem. 84(18), 7845-7851 (2012).

19. A. Lattermann et al., "Characterization of atherosclerotic plaque depositions by Raman and FTIR imaging," J. Biophotonics 6(1), 110-121 (2013).

20. A. Phinikaridou et al., "A robust rabbit model of human atherosclerosis and atherothrombosis," J. Lipid Res. 50(5), 787-797 (2009).

21. U. Speck et al., "How does a drug-coated balloon work? Overview about coating technologies and their impact," J. Cardiovasc. Surg. 57(1), 3-11 (2016).

22. T. Zeller et al., "Novel approaches to the management of advanced peripheral artery disease: perspectives on drug-coated balloons, drugeluting stents, and bioresorbable scaffold," Curr. Cardiol. Rep. 17(9), 624 (2015).

23. S. Banerjee et al., "Drug-coated balloon and stent therapies for endovascular treatment of atherosclerotic superficial femoral artery disease," Curr. Cardiol. Rep. 17(5), 36 (2015).

24. K. Balss et al., "Multivariate analysis applied to the study of spatial distributions found in drug-eluting stent coatings by confocal Raman microscopy," Anal. Chem. 80(13), 4853-4859 (2008).

25. A. Belu, C. Mahoney, and K. Wormuth, "Chemical imaging of drug eluting coatings: combining surface analysis and confocal Raman microscopy," J. Controlled Release 126(2), 111-121 (2008).

26. I. Schie et al., "Direct comparison of fatty acid ratios in single cellular lipid droplets as determined by comparative Raman spectroscopy and gas chromatography," Analyst 138(21), 6662-6670 (2013).

27. ssniff ${ }^{\circledR}$, ssniff Produkt Katalog, p. 58, ssniff Spezialdiäten GmbH, Soest, Germany (2015).

28. C. Matthäus et al., "Multimodal nonlinear imaging of atherosclerotic plaques differentiation of triglyceride and cholesterol deposits," J. Innovative Opt. Health Sci. 7(5), 1450027 (2014).

29. T. Wang et al., "Detection of plaque structure and composition using OCT combined with two-photon luminescence (TPL) imaging," Lasers Surg. Med. 47(6), 485-494 (2015).
30. J. Phipps et al., "A fluorescence lifetime imaging classification method to investigate the collagen to lipid ratio in fibrous caps of atherosclerotic plaque," Lasers Surg. Med. 44(12), 564-571 (2012).

31. R. Cicchi et al., "Non-linear imaging and characterization of atherosclerotic arterial tissue using combined SHG and FLIM microscopy," J. Biophotonics 8(4), 347-356 (2015).

32. A. Ko et al., "Multimodal nonlinear optical imaging of atherosclerotic plaque development in myocardial infarction-prone rabbits," J. Biomed. Opt. 15(2), 020501 (2010).

33. R. Lim et al., "Multimodal CARS microscopy determination of the impact of diet on macrophage infiltration and lipid accumulation on plaque formation in ApoE-deficient mice," J. Lipid Res. 51(7), 1729-1737 (2010).

Christian Matthäus is working as a research assistant at Leibniz Institute for Photonic Technology (IPHT), Jena, Germany. His current interests are Raman spectroscopy, nonlinear imaging techniques, and MALDI-TOF. He received his PhD at City University of New York in 2006 in the field of spectroscopic imaging. After a postdoc position at Northeastern University, Boston, he joined the group of Prof. Jürgen Popp at the IPHT in 2009.

Sebastian Dochow received his PhD in 2013 at Friedrich Schiller University (FSU), Jena in the group of Prof. Jürgen Popp. Later, he worked as a postdoc at FSU and IPHT. He is currently employed as a technical engineer at Jena-Optronic.

Kokila D. Egodage has been a PhD student at Friedrich Schiller University Jena, Germany. Her research work on the combination of optical coherence tomography and Raman spectroscopy focused on discerning diseased biological tissue. After graduating from the University of New South Wales Sydney, Australia, she was involved in a chip-based research project at the Technical University of Eindhoven, the Netherlands. Currently, she is based at Carl Zeiss SMT GmbH, Jena, as an application engineer.

Bernd F. Romeike is responsible for the neuropathological section at Jena Mecical School, Germany, and clinical neuropathologist. He received his MD in Frankfurt am Main, Habilitation at the Homburg Medical School and the master of medical education in Heidelberg, Germany. His active research interests include the application of intravital optical microscopy techniques in clinical settings. He has over 20 years of experience performing and directing biomedical research in neuropathology.

Bernhardt R. Brehm received his PhD from Ruprecht-KarlsUniversity Heidelberg. After working as a cardiologist in Thübingen, Jena, and Koblenz, he moved to the Heart-Neuro-Center in Kreuzlingen, Switzerland, where he is currently working as a chief physician in cardiology.

Jürgen Popp studied chemistry at the Universities of Erlangen and Würzburg. After his PhD in chemistry, he joined Yale University for postdoctoral work. Since 2002 he holds a chair for physical chemistry at Friedrich-Schiller University Jena. Since 2006, he is also the scientific director at the Institute of Photonic Technology, Jena. His research interests are mainly concerned with innovative Raman techniques and biophotonics. 